\title{
Ionic Matrix for Matrix-Enhanced Surface-Assisted Laser Desorption Ionization Mass Spectrometry Imaging (ME-SALDI-MSI)
}

\author{
Qiang Liu and Lin He \\ Department of Chemistry, North Carolina State University, Raleigh, North Carolina, USA
}

\begin{abstract}
Matrix-enhanced surface-assisted laser desorption ionization mass spectrometry imaging (ME-SALDI MSI) has been previously demonstrated as a viable approach to improving MS imaging sensitivity. We describe here the employment of ionic matrices to replace conventional MALDI matrices as the coating layer with the aims of reducing analyte redistribution during sample preparation and improving matrix vacuum stability during imaging. In this study, CHCA/ANI ( $\alpha$-cyano-4-hydroxycinnamic acid/aniline) was deposited atop tissue samples through sublimation to eliminate redistribution of analytes of interest on the tissue surface. The resulting film was visually homogeneous under an optical microscope. Excellent vacuum stability of the ionic matrix was quantitatively compared with the conventional matrix. The subsequently improved ionization efficiency of the analytes over traditional MALDI was demonstrated. The benefits of using the ionic matrix in MS imaging were apparent in the analysis of garlic tissue sections in the ME-SALDI MSI mode. (J Am Soc Mass Spectrom 2009, 20, 2229-2237) (C) 2009 American Society for Mass Spectrometry
\end{abstract}

S urface-assisted laser desorption ionization mass spectrometry (SALDI-MS) has been used as a complementary tool to matrix-assisted laser desorption ionization mass spectrometry (MALDI-MS) in small molecule profiling $[1,2]$. Its applications in tissue imaging have been demonstrated by various groups where porous surfaces or inorganic nanoparticles have been successfully utilized as the energy mediating reagents [3-5]. To further improve imaging sensitivity, a hybrid ionization approach, matrix-enhanced SALDI (ME-SALDI), has been recently reported in which conventional matrix molecules were deposited atop a porous SALDI substrate to provide a proton-rich environment for enhanced ionization of desorbed species [6]. The porous SALDI substrate in ME-SALDI is believed to effectively reduce laser flux needed for analyte desorption; consequently few matrix ions and fragments are observed in the resulting spectra. Both characteristics are crucial for detection of low-mass species, rendering ME-SALDI an attractive solution for MS imaging (MSI) of metabolites, especially for those where imaging at high spatial resolution under a reduced laser beam size is desired. Improved MS performance over conventional MALDI and SALDI ionization methods has been demonstrated with enhanced detection sensitivity and a broadened detection mass window [6].

Address reprint requests to Professor Lin He, Department of Chemistry, North Carolina State University, Raleigh, NC 27695, USA. E-mail: lin_he@ ncsu.edu
However, an inherent challenge associated with matrix-based laser desorption ionization sources is also present in ME-SALDI for MSI applications: it is known that most conventional MALDI matrices slowly vaporize under high vacuum $\left(10^{-7} \sim 10^{-8}\right.$ Torr $)$ due to their low sublimation points. The loss of materials becomes more severe when the matrix is amorphous-a common form observed when the matrix is deposited by sublimation in a solvent-free fashion. While the loss of matrix under vacuum does not necessarily impose a problem in MALDI profiling experiments where most measurements occur in seconds, it cannot be ignored during MS imaging experiments because of extended interrogation time and the preferred solvent-free approach for matrix deposition [7]. For example, for a commercial MALDI-TOF instrument equipped with a $20-\mathrm{Hz} \mathrm{N} \mathrm{N}_{2}$ laser, approximately 720 pixels can be imaged in $1 \mathrm{~h}$ when 100 laser shots are averaged per pixel, which translates to $\sim 55.6 \mathrm{~h}$ of instrument time to examine a $1-\mathrm{cm}^{2}$ tissue section with spatial resolution of $50 \mu \mathrm{m}$, not counting the time spent for translational stage movement. Even with a high pulse rate laser installed, the interrogation time for most imaging experiments is sufficiently long to experience the impacts of matrix loss, most notably the inevitable change in ionization efficiency across tissue that skews the actual spatial distribution of chemical composition in the sample imaged.

A new class of MALDI matrices by mixing an equal molar of a conventional MALDI matrix with an organic base to form the so-called ionic matrix have been reported 
by Armstrong et al. in 2001, [8] and the progress to date has been summarized in a recent review by Tholey and Heinzle [9]. In comparison with conventional MALDI matrices, ionic matrices afford several advantages, including improved deposition homogeneity, suppressed matrix background interference, reduced matrix fragmentation, and more importantly for imaging applications, superior stability under high vacuum over an extended MS inquisition period. Many types of ionic matrices have since been synthesized and evaluated. Their applications in detection and quantification of oligonucleotides, lipids, and saccharides have gained great research interest [10-21]. Direct tissue analysis in the MALDI mode using ionic matrices has shown better spectral quality and ionization efficiency over their conventional analogs [20, 21]. MS imaging using ionic matrices in the conventional MALDI mode has also been reported [20].

Here, we describe the use of an ionic matrix, i.e., CHCA/ ANI synthesized with $\alpha$-cyano-4-hydroxycinnamic acid (CHCA) and aniline (ANI), in the place of conventional MALDI matrix, for ME-SALDI MS imaging. CHCA/ ANI was selected as the model for their demonstrated performance in conventional MALDI MS imaging [9, 20]. In this study, reproducible and homogenous deposition of CHCA/ANI through sublimation has been demonstrated, which eliminates solvent-induced analyte dissolution and diffusion on the tissue surface. Spectral qualities in terms of detectable mass range and mass resolution of ionic matrix-based ME-SALDI are comparable to those of MALDI using ionic matrices. Similarly improved MS performance was observed when CHCA/pyridine was used as another ionic matrix model. Reduced spectral background and better MS ionization efficiency of ionic matrix-based ME-SALDI over ionic matrix-based MALDI renders it an ideal solution for 2-D metabolite imaging.

\section{Experimental}

\section{Materials}

N-type Sb-doped (100) single-crystalline silicon wafers at $0.005-0.02 \Omega / \mathrm{cm}$ were purchased from Silicon Sense, Inc. (Nashua, NH, USA), and stored under vacuum. 2,5Dihydroxybenzoic acid (DHB), $\alpha$-cyano-4-hydroxycinnamic acid (CHCA), quinidine, pyridine (Py), and aniline (ANI) were purchased from Sigma Aldrich (St. Louis, MO, USA). Methylene blue $\left(\mathrm{C}_{18} \mathrm{H}_{22} \mathrm{~N}_{3} \mathrm{SCl} \cdot 1 / 2 \mathrm{ZnCl}_{2}\right)$ was purchased from MP Biomedicals (Solon, $\mathrm{OH}, \mathrm{USA}$ ). Hydrofluoric acid $(\mathrm{HF}, 49 \%)$, acetone, methanol, and $\mathrm{H}_{2} \mathrm{O}_{2}(30 \%)$ were purchased from Fisher Scientific (Pittsburgh, PA, USA). $\mathrm{CH}_{3} \mathrm{CH}_{2} \mathrm{OH}$ was purchased from Aaper Alcohol (Shelbyville, KY, USA). DI $\mathrm{H}_{2} \mathrm{O}$ of $18 \mathrm{M} \Omega$ (Millipore, Danvers, MA, USA) was used throughout the experiments. Mouse brain and chicken liver tissues were freshly frozen and stored at $-80{ }^{\circ} \mathrm{C}$ upon use. Garlic and chicken liver tissue samples were purchased from local grocery stores.

\section{Porous Silicon Substrate Preparation}

Porous silicon substrates were prepared as previously described [22]. Briefly, silicon wafer chips were washed with a $5 \%$ HF solution in ethanol for $1 \mathrm{~min}$ and then electrochemically etched in a $25 \% \mathrm{HF}$ /ethanol solution for $1 \mathrm{~min}$ at a current density of $5 \mathrm{~mA} / \mathrm{cm}^{2}$. White light from a $50 \mathrm{~W}$ tungsten lamp was used to irradiate the silicon surface during etching. The produced porous silicon substrates were then dipped into $15 \% \mathrm{H}_{2} \mathrm{O}_{2}$ for 1 min, followed by a 1-min dip in 5\% HF/ethanol solution to refresh the surface before analyte deposition.

\section{Synthesis of Ionic Matrix}

CHCA/ANI and CHCA/Py were synthesized by mixing an equal molar CHCA with ANI (or Py) in methanol as described in the literature [20]. The reaction mixture was stirred for $2 \mathrm{~h}$ at room temperature, followed by solvent removal under vacuum. The synthesized products, CHCA/ANI and CHCA/Py, were stored in a desiccator under vacuum at room temperature before use.

\section{MS Sample Preparation}

Two standard solutions of quinidine were prepared for MS evaluation of ionic matrix-based ME-SALDI: (1) $1-\mu \mathrm{M}$ quinidine in $4.2 \mathrm{mg} / \mathrm{mL}$ of $\mathrm{CHCA} / \mathrm{ANI}$ in acetonitrile/water (1:1, vol/vol), and (2) $1-\mu \mathrm{M}$ quinidine in $4.2 \mathrm{mg} / \mathrm{mL}$ of CHCA/ANI in acetonitrile/water (1:1, vol/vol, containing $0.1 \%$ TFA). For MALDI-MS analysis, $1 \mu \mathrm{L}$ of the standard solution (1) or (2) was droploaded on a stainless steel MALDI plate. For ME-SALDI analysis, $1 \mu \mathrm{L}$ of standard solution (1) was drop-loaded on a porous silicon substrate.

To study the impact of acidic additives on vacuum stability, two $10 \mathrm{mg} / \mathrm{mL} \mathrm{CHCA} / \mathrm{ANI}$ solutions were prepared in acetone:ethanol $=2: 1$ with or without $0.1 \%$ TFA. Individual glass slides were placed on a Laurell WS-400E-6NPP-LITE spin coater (North Wales, PA, USA), followed by dropping $50 \mu \mathrm{L}$ of the CHCA/ANI solution with or without TFA to the center of the slides. The spin coater was then operated at $300 \mathrm{rpm}$ for $1 \mathrm{~min}$ that yielded a uniform matrix film.

Tissue samples were prepared by first mounting sections of garlic cloves or chicken liver on a metal support with an optical cutting temperature (OCT) compound. The mounted species were then sliced into $10-\mu \mathrm{m}$ thick sections with a Cryo-cut microtome (American Optical Corp., Buffalo, NY, USA) at $-20^{\circ} \mathrm{C}$. The UV absorbance of chicken liver and garlic tissue slices was measured to examine the transparency of the resulting tissue samples using an 8453 Hewlett Packard spectrometer (Agilent, Palo Alto, CA, USA) (Supporting Information SF $1 a$ and $b$, which can be found in the electronic version of this article). The sliced sections were carefully transferred onto a porous silicon substrate for ME-SALDI MSI. Meanwhile, a tissue section 
microtomed at the adjacent location was placed on a glass slide, stained with methylene blue, and imaged with a Leica DMRX light microscope (Leica Microsystems $\mathrm{GmbH}$, Germany). In some cases, limited by the view of microscope, the optical images of stained sections were mosaics of several individual images.

Matrix deposition for imaging applications was carried out by sublimation. Specifically, $0.3 \mathrm{~g}$ of $\mathrm{CHCA}$, $\mathrm{CHCA} / \mathrm{ANI}, \mathrm{CHCA} / \mathrm{Py}$, or $\mathrm{DHB}$ was added into a sublimation chamber set up in-house [7]. A tissuecoated porous silicon substrate or a transparent glass slide was mounted to the flat bottom of the condenser, facing downwards to the matrix. An oil bath was preheated to $120^{\circ} \mathrm{C}$ for $\mathrm{CHCA}, 170{ }^{\circ} \mathrm{C}$ for $\mathrm{CHCA} / \mathrm{ANI}$, $113^{\circ} \mathrm{C}$ for $\mathrm{CHCA} / \mathrm{Py}$, or $110^{\circ} \mathrm{C}$ for $\mathrm{DHB}$ deposition. The amount of matrix deposited was controlled by the sublimation time or the temperature of the oil bath, which was varied from $80^{\circ} \mathrm{C}$ to $120^{\circ} \mathrm{C}$. The condenser and the surface temperature of the substrate attached to the condenser were cooled down with running water for vapor condensation. The pressure of the sublimation chamber was maintained at $\sim 50$ Torr for $2 \mathrm{~min}$ before immersing the apparatus into the oil bath. Except for $\mathrm{CHCA} / \mathrm{Py}$, the sublimation setup was removed and the vacuum was released after 5 -min immersion. A $10-\mathrm{min}$ heating was used in the sublimation of $\mathrm{CHCA} / \mathrm{Py}$. The substrate was then carefully removed, and the amount of matrix deposited was subsequently quantified by UV absorbance measurements of the matrix-coated glass slides. Deposition uniformity was evaluated by calculating intra-substrate relative standard deviations of the measured UV absorbance of five different locations on the same substrate. Deposition reproducibility was evaluated by inter-substrate relative standard deviations, which were calculated from UV absorbance of five matrix-coated glass sides prepared under same conditions. Optical microscopic images were also taken to visually examine the quality of matrix deposition using a Leica DMRX light microscope equipped with a Donpisha XC-003P CCD camera. Matrix stability was evaluated by comparing UV absorbance of matrices before and after storage in the sample chamber of the mass spectrometer $\left(10^{-7}\right.$ Torr) for $40 \mathrm{~h}$.

An adjacent slice of tissue from the same garlic clove used in MS imaging was homogenized and extracted in methanol under stirring for $2 \mathrm{~d}$. CHCA/ ANI $(4.2 \mathrm{mg} / \mathrm{mL})$ was then added to the filtered garlic extracts before being drop-deposited on a substrate for ME-SALDI and MALDI profiling measurements. For the experiments where $\mathrm{CHCA} / \mathrm{Py}$ was used, one volume of garlic methanol extract was mixed with one volume of $6 \mathrm{mg} / \mathrm{mL}$ of CHCA aqueous solution or $8 \mathrm{mg} / \mathrm{mL}$ of CHCA/Py aqueous solution for MALDI and ME-SALDI experiments, respectively. In LDI and SALDI measurements, the garlic extract was diluted with one volume of water instead of with the matrix solution.

\section{MS Instrument Parameters and Data Analysis}

An Applied Biosystems Voyager DE-STR MALDI-TOF mass spectrometer (Framingham, MA, USA) was used for most experiments, which was operated at an accelerating voltage of $20 \mathrm{kV}$, equipped with a $20-\mathrm{Hz} \mathrm{N}_{2}$ laser. All experiments were operated in reflector mode. The laser intensity and delay time were varied to achieve optimal performance. An adjustable iris was used to change the diameter of the laser beam, and the final beam size was determined by the largest measurable laser burn-mark left on a substrate under the highest laser fluence provided by the instrument [5]. A $35-\mu \mathrm{m}$ laser beam was used in most experiments, except for DHB-coated garlic section and CHCA-coated mouse brain section, which were imaged under a $90-\mu \mathrm{m}$ laser beam at $100-\mu \mathrm{m}$ stepwise to acquire appreciable signals [6]. CHCA/ANI-coated garlic section was imaged at 50- $\mu \mathrm{m}$ stepwise.

For the experiments where MS performances of MALDI, LDI, SALDI, and ME-SALDI were compared, the data were collected with a Bruker Daltonics (Billerica, MA, USA) Omniflex MALDI-TOF MS instrument. The signal-to-noise ratios were calculated with XMASS 5.1.

Mass spectra were extracted using in-house developed software and plotted using Origin 6.0 (OriginLab Corp., Northampton, MA, USA). Matrix suppression effect scores (MSE) were calculated using MSE $=\frac{\sum \mathrm{I}_{[\text {Analyte Ions }]}}{\sum \mathrm{I}_{\text {[Analyte Ions }]}+\sum \mathrm{I}_{[\text {Matrix Ions }]}}$ to quantify the impact of matrix interference. During MS imaging, the instrument was controlled by MMSIT MALDI Imaging Tool software v2.2.0. The final ion maps, as well as the $\mathrm{Y}$-axis and $\mathrm{X}$-axis profiles, were reconstructed using BioMap 3.7.5.4. Both programs were provided by Dr. Markus Stoeckli, Novartis Institutes for BioMedical Research, Basel, Switzerland.

\section{Results and Discussion}

The gradual loss of matrix molecules in MS imaging was clear in Figure 1a and b, where conventional matrix DHB was sublimated atop a piece of garlic tissue section. DHB was chosen as the representative matrix for conventional MALDI in this study for its popularity in MSI applications and the better vacuum stability of its amorphous film than other widely used conventional MALDI matrices. Panel 1a shows the clearly reduced molecular ion intensity of DHB $(m / z=155.0)$ over $\sim 15 \mathrm{~h}$ scanning, following the overall rastering direction from left to right. Similarly skewed distribution of analyte ions is also observed. As shown in Figure $1 b$, a much higher ion intensity for the ion at $m / z 104.1$, likely from $\gamma$-aminobutyric acid, one of the major amino compounds produced during germination and early seedling growth of plant $[23,24]$, is observed not only in the cotyledon portion of garlic, as expected, but also in the left portion of the tissue where the area was ana- 

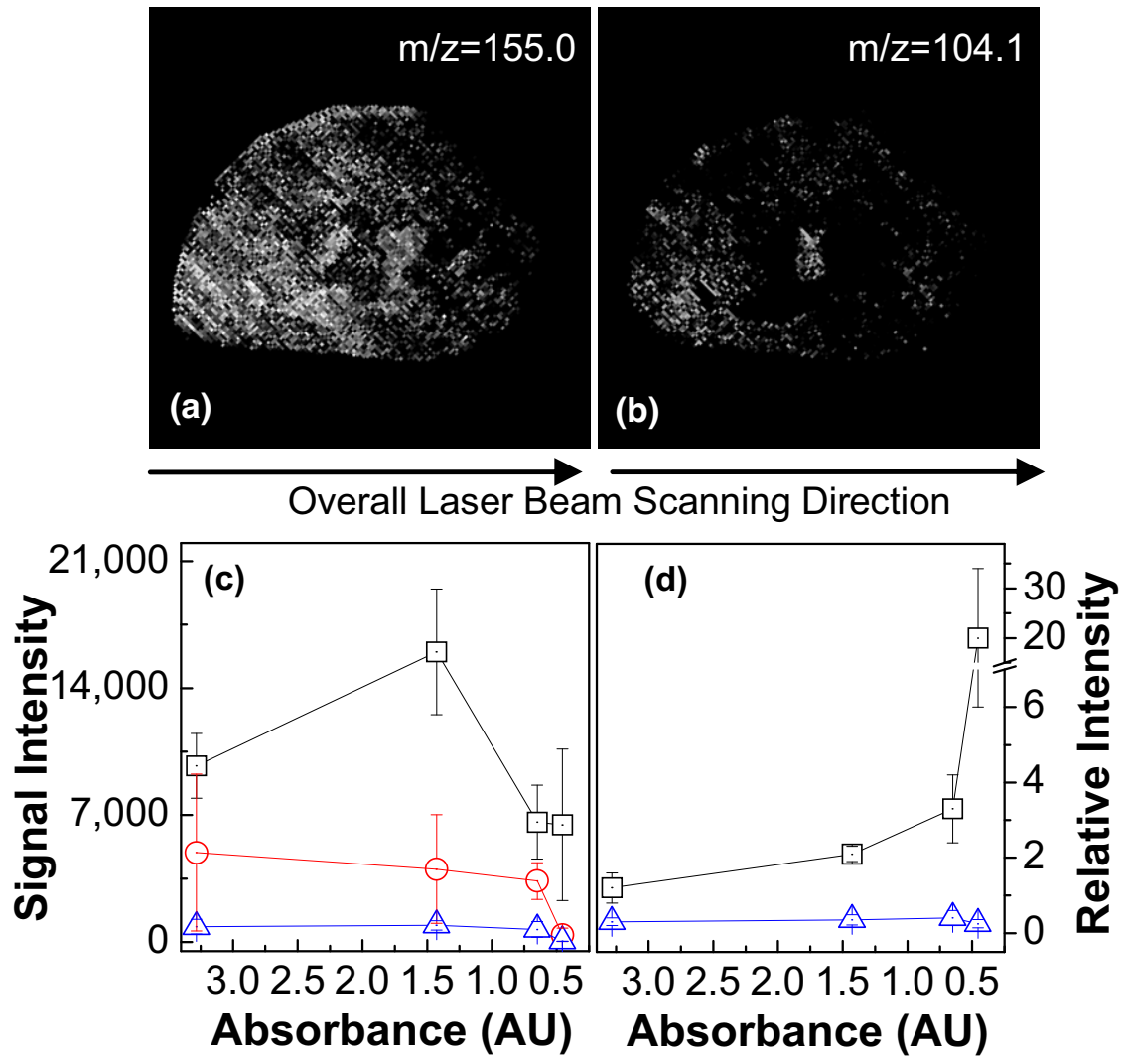

Figure 1. (Top) Reconstructed 2-D MS images for ions at (a) $\mathrm{m} / \mathrm{z}=155.0$ and (b) $\mathrm{m} / \mathrm{z}=104.1$ for DHB-coated garlic tissue. (Bottom) DHB was sublimated onto both chicken liver tissue section-coated porous $\mathrm{Si}$ substrates and transparent glass slides with varied thicknesses to investigate the impact of different amount of DHB in MS detection. (c) A plot of MS signal intensities of an analyte ion at $\mathrm{m} / \mathrm{z}=$ 103.9 (open square), the matrix ion at $\mathrm{m} / \mathrm{z}=155.0$ (open circle), and a major cholesterol ion at $\mathrm{m} / \mathrm{z}=$ 369.4 (open triangle) against the absorbance of DHB on the glass slides of the same DHB thickness at $337 \mathrm{~nm}$. (d) Normalized MS ion intensities at $\mathrm{m} / \mathrm{z}=103.9$ (open square) and $\mathrm{m} / \mathrm{z}=369.4$ (open triangle) against the DHB molecular ions at $m / z=155.0$ as a function of the absorbance of DHB at $337 \mathrm{~nm}$.

lyzed first during the MSI experiment. Note that the garlic tissue as well as the chicken liver tissue used in the subsequent studies merely served as the sample tissue to reveal the improved MS performance when ionic matrices were used. In-depth understanding of its corresponding biologic contents and optimization of sample preparation is ongoing.

To better quantitate the effect of matrix loss on MS imaging, MS spectra were collected from several adjacent chicken liver tissue sections that were placed on porous Si MS substrates and were coated with different amounts of DHB by controlling the sublimation temperature (Supporting Information Figure 1). Meanwhile, for each tissue-coated MS substrate, a tissuecoated glass slide was prepared at the same time in the same chamber to ensure consistent coating of DHB on the surface. The transmission UV absorbance measurements show that the typical amount of matrix used in ME-SALDI experiments was sufficiently low, which allowed photons to reach the porous substrate underneath even with tissue samples lying on the top (Supporting Information Figure $1 \mathrm{~b}$ and $\mathrm{c}$ ). The MS ion intensities of several prominent species acquired from the chicken liver tissue were plotted against the UV absorbance of DHB at $337 \mathrm{~nm}$ from the corresponding glass slides. Clearly, the MS intensities of analyte ions vary when the amount of DHB changes and the degree of variation differs significantly among the analytes studied (Figure 1c). For example, the major ion peaks of DHB $\left([\mathrm{M}+\mathrm{H}]^{+}, m / z\right.$ 155.0) and cholesterol ([M + H $\left.\mathrm{H}_{2} \mathrm{O}\right]^{+}, m / z$ 369.4) remained relatively constant initially but decreased sharply when the amount of matrix deposited reduced below certain thresholds. In contrast, the intensity of the ions at $\mathrm{m} / \mathrm{z} 103.9$ increased initially as the thickness of DHB decreased but decreased rapidly when the amount of DHB was less than 1.5 AU. The observation confirmed our initial concern on the possible distortion of MS imaging results due to the loss of matrix across the surface that was slowly pumped away under vacuum. This nonuniformity in matrix distribution could impose an upper limit to the MS imaging inquisition time, which directly translates to the limits on the overall size of the tissue section to be analyzed and on the achievable spatial resolution when conventional MALDI matrices are used in MS imaging. It is also worth pointing out that internal standards 
have been used in the literature to alleviate the distortion induced by the loss of matrix over time. However, the fact that the degree of matrix-induced MS signal fluctuation is closely dependent on the chemical nature of the analyte to be imaged makes this approach only partially successful. As shown in Figure 1d, after being normalized against the molecular ion peak of DHB, the ion intensities of cholesterol became less dependent on the amount of DHB on the substrate, where the relative signal variation of cholesterol ions across the samples with different amounts of matrix was reduced from $63 \%$ to $20 \%$. However, a sharp increase in the relative intensity was observed for the ion at $\mathrm{m} / \mathrm{z} 103.9$ after DHB normalization, and the calculated signal variation jumped from $46 \%$ to $134 \%$.

As previously discussed, advances in matrix optimization suggest ionic matrix as a viable solution to the aforementioned problem. Before it can be employed in ME-SALDI MS imaging, however, three critical issues need to be addressed first to ensure a homogenous and reproducible matrix coating with minimal analyte delocalization: (1) deposition of ionic matrix can be conducted in a solvent-free format, e.g., through sublimation; (2) matrix stability is indeed improved in the ME-SALDI mode that warrants continuous investigation and optimization; and (3) the spectral integrity is not compromised in ME-SALDI when ionic matrix is used. To address these issues, an ionic matrix, CHCA/ ANI, was selected as the model complex to evaluate the performance of ionic matrix in ME-SALDI for its demonstrated superior MALDI performance in tissue imaging applications [20]. Note that because the ionic matrix in ME-SALDI solely serves as a proton-rich donor to enhance ionization efficiency, most ionic matrices that have been demonstrated viable for conventional MALDI should work in a similar fashion in ME-SALDI.

Although in salt forms, many ionic liquid molecules exhibit measurable vapor pressures that allow them to be vaporized at reduced pressure without decomposition [25]. Indeed, CHCA/ANI was found to be vaporizable at an elevated temperature of $170^{\circ} \mathrm{C}$, noticeably higher than that of $\mathrm{CHCA}$ under vacuum $\left(125^{\circ} \mathrm{C}\right)$, but still manageable with the in-house sublimation apparatus. As shown in Figure 2a, homogenous matrix films with small crystal size $(\sim 1 \mu \mathrm{m})$ were produced through sublimation. A uniform layer of CHCA-Py has also been produced through sublimation (Supporting Information Figure 2a). Dissociation of CHCA/ANI and $\mathrm{CHCA} / \mathrm{Py}$ salt were unlikely during vaporization, as drastically different film morphology was observed from the CHCA/ANI or CHCA/Py-coated slide in comparison with the surface coated with CHCA alone (Supporting Information Figure 2b).

To quantitatively assess the reproducibility and stability of matrix deposition, the corresponding UV absorbance from CHCA/ANI- and DHB-coated glass slides was compared. The intra-substrate RSD\% of five
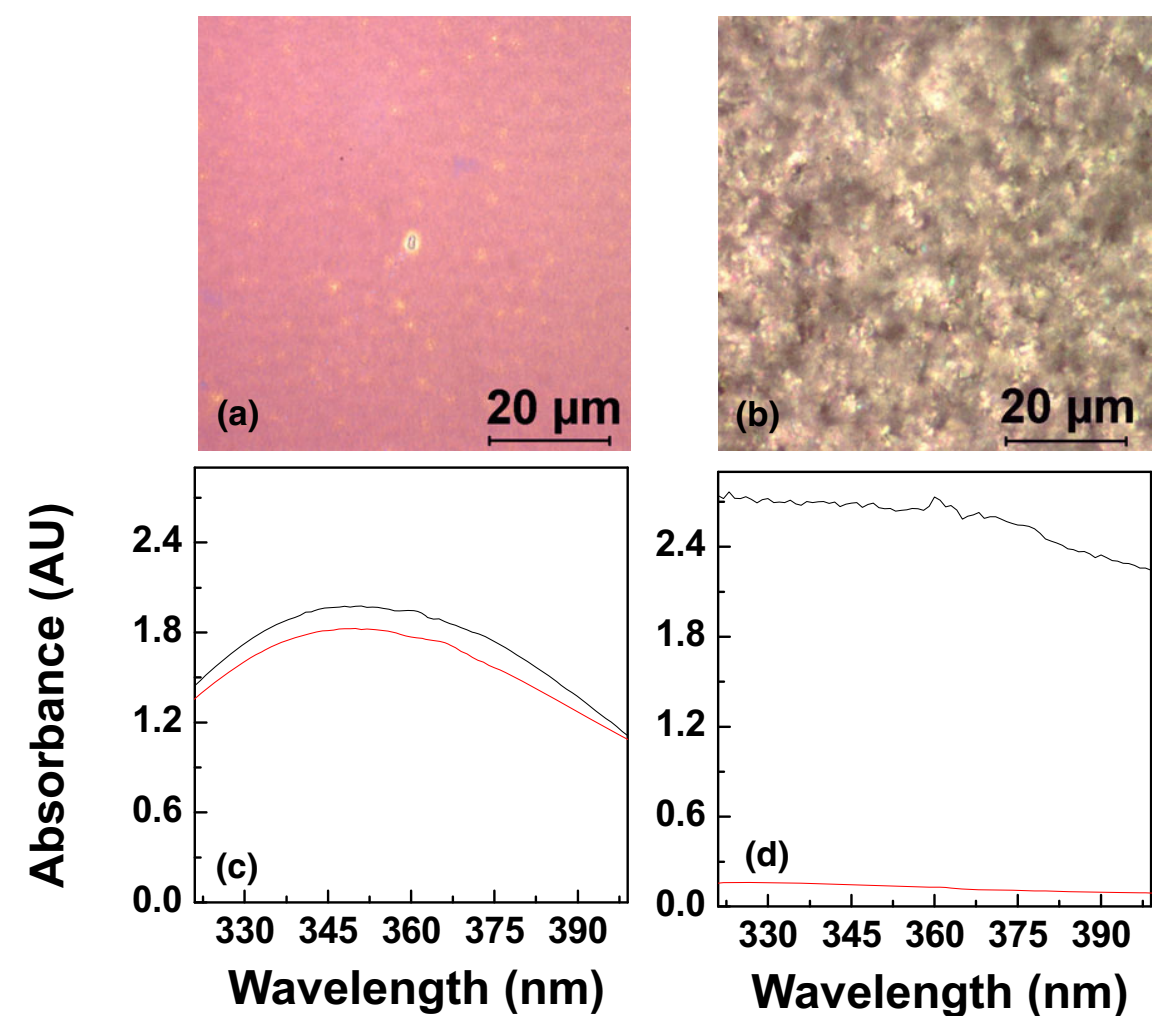

Figure 2. Optical images of (a) the solid ionic matrix (CHCA/ANI) and (b) conventional matrix (DHB) sublimated on transparent glass slides. Representative UV-Vis spectra of (c) the CHCA/ANI layer and (d) the DHB layer collected before (black) and after (red) 40-h vacuum storage. 
Table 1. Signal-to-noise ratios of represent analytes detected in garlic extract using CHCA and CHCA/Py as the matrices in MALDI, using direct laser desorption ionization (LDI), using a porous silicon substrate in SALDI, and using CHCA/Py on a porous Si substrate in ME-SALDI

\begin{tabular}{|c|c|c|c|c|c|}
\hline \multirow[b]{2}{*}{ Analyte } & \multicolumn{2}{|c|}{ MALDI } & \multirow{2}{*}{$\begin{array}{c}\text { LDI } \\
\text { No matrix }\end{array}$} & \multirow{2}{*}{$\begin{array}{c}\text { SALDI } \\
\text { No matrix }\end{array}$} & \multirow{2}{*}{$\begin{array}{l}\text { ME-SALDI } \\
\text { CHCA-Py }\end{array}$} \\
\hline & $\mathrm{CHCA}$ & CHCA-Py & & & \\
\hline$\gamma$-Aminobutyric acid $(m / z=104.1)$ & $1425 \pm 384$ & $4126 \pm 2478$ & $886 \pm 1280$ & $4773 \pm 2658$ & $61072 \pm 28791$ \\
\hline Unknown ion $(m / z=796.5)$ & $551 \pm 97$ & $646 \pm 342$ & NA & $158 \pm 107$ & $21038 \pm 9061$ \\
\hline $\operatorname{DP6}(m / z=1029.3)$ & $69 \pm 17$ & NA & NA & NA & $2793 \pm 1698$ \\
\hline $\operatorname{DP9}(m / z=1515.4)$ & $40 \pm 9$ & NA & NA & NA & $961 \pm 429$ \\
\hline $\operatorname{DP} 12(m / z=2001.6)$ & $14 \pm 2$ & NA & NA & NA & $231 \pm 97$ \\
\hline
\end{tabular}

$\mathrm{NA}=$ no ion peak discernable.

measurements within the same substrate coated with CHCA/ANI was less than $2 \%$ and the inter-substrate $\mathrm{RSD} \%$ from five CHCA/ANI-coated substrates prepared independently was less than $4.4 \%$ (Supporting Information Table 1). On the other hand, for DHBcoated substrates the intra-substrate and inter-substrate RSD of $3 \%$ and $8.2 \%$ was calculated, respectively. The results suggest a comparable, if not better, deposition uniformity and reproducibility when ionic matrix was deposited by sublimation. The vacuum stability of different matrix layers was examined by comparing the change in their UV absorbance before and after 40-h vacuum storage at $10^{-7}$ Torr. As shown in Figure 2c, the UV absorbance of the CHCA/ANI layer changed less than $4 \%$ (from $1.81 \pm 0.05$ to $1.75 \pm 0.03$ ). In contrast, the UV absorbance of the substrate coated with DHB was significantly reduced from $2.68 \pm 0.05$ to $0.15 \pm$ 0.02 , equivalent to more than $94 \%$ loss of the original materials (Figure 2d).

Significant reduction of the matrix interference is another attractive characteristic of using CHCA/ANI as the matrix, yet it was often accompanied by decrease of analyte signals. As shown in Figure 3a, a clean but fairly weak analyte signal was observed when CHCA/ANI was used as the MALDI matrix in detection of 1 pmol quinidine $(m / z=325.2)$, an anti-arrhythmic pharmaceutical compound. The Matrix Suppression Effect score $\left(\right.$ MSE $\left.=\frac{\sum \mathrm{I}_{[\text {Analyte Ions }]}}{\sum \mathrm{I}_{[\text {Analyte Ions }]}+\sum \mathrm{I}_{[\text {Matrix Ions }]}}\right)$ was quantitatively calculated as $72 \pm 7 \%$. Proton transfer between matrix ions and analyte molecules in desorbed plume is believed to be one of the primary ionization pathways for analyte ionization in MALDI. Decrease of the plume density for the ionic matrix system, i.e., fewer gas-phase inter-molecular collisions, is suspected, which likely results in lower yields of analyte and matrix ions. Furthermore, the less acidic nature of ionic matrices than that of conventional organic matrices offers fewer protons to the desorbed species, which further reduces the ionization efficiency of the system. Acidic additives, such as trifluoroacetic acid (TFA) or phosphoric acid, have been frequently used with ionic matrices in MALDI-MS to overcome this limitation and enhance analyte ionization efficiency (Figure 3b). However, these acidic additives cannot be co-vaporized with $\mathrm{CHCA} /$
ANI due to their drastically different vapor pressures; therefore solution-based matrix deposition has to be employed, which raises the risks of analyte redistribution. More importantly, although these acidic additives facilitate analyte ionization by providing extra protons to the desorbed species, they compete with CHCA in forming complexes with aniline, resulting in release of conventional MALDI matrix molecules from the corresponding solid ionic matrix (Supporting Information Scheme 1). Reappearance of the matrix signals after addition of TFA was the first sign of dissociation of CHCA/ANI complexes (Figure 3b), substantiated by the decreased MSE value to $21 \pm 5 \%$. Suspected dissociation was further confirmed by the reduced vacuum stability where the UV absorbance of CHCA/ANI codeposited with TFA decreased from $0.73 \pm 0.16$ to $0.50 \pm 0.11$ after 40 -h storage. It corresponds to a $32 \%$ reduction, a more than 8 -fold increase in the matrix loss rate compared with that of $\mathrm{CHCA} / \mathrm{ANI}$ without TFA (data not shown).

Using porous surfaces to assist efficient ionization of analytes, ME-SALDI provides an alternative solution to improve analyte signals by enhancing the desorption efficiency. Figure $3 c$ shows that the ion intensity of quinidine was improved for nearly two orders of magnitude in ME-SALDI in comparison with using the same ionic matrix in the MALDI mode without TFA, and more than 3-fold to that in the MALDI mode with TFA. Moreover, the desired characteristics of ionic matrices in reducing matrix interference (MSE $=69 \pm 5 \%$ ) and improving vacuum stability (less than $4 \%$ loss after $40-\mathrm{h}$ vacuum storage) were preserved in ME-SALDI.

The benefit of combining porous substrates with ionic matrix in the ME-SALDI mode was also demonstrated in the detection of garlic extracts. The ion intensity at $m / z 104.1$ was $\sim 7$-fold stronger in ME-SALDI (Figure 4a) than in MALDI without acidic additives (Figure 4b). The molecular ions of fructo-oligosaccharides with the degree of polymerization (DP) from 5 to 12 were unambiguously detected only in ME-SALDI. Furthermore, the matrix background signal was negligible. Last but not the least, other important MS characteristics, such as absolute ion intensity, signal-to-noise ratio, mass range, and spectral resolution of the analytes, were preserved, if not improved, when the ionic matrix 

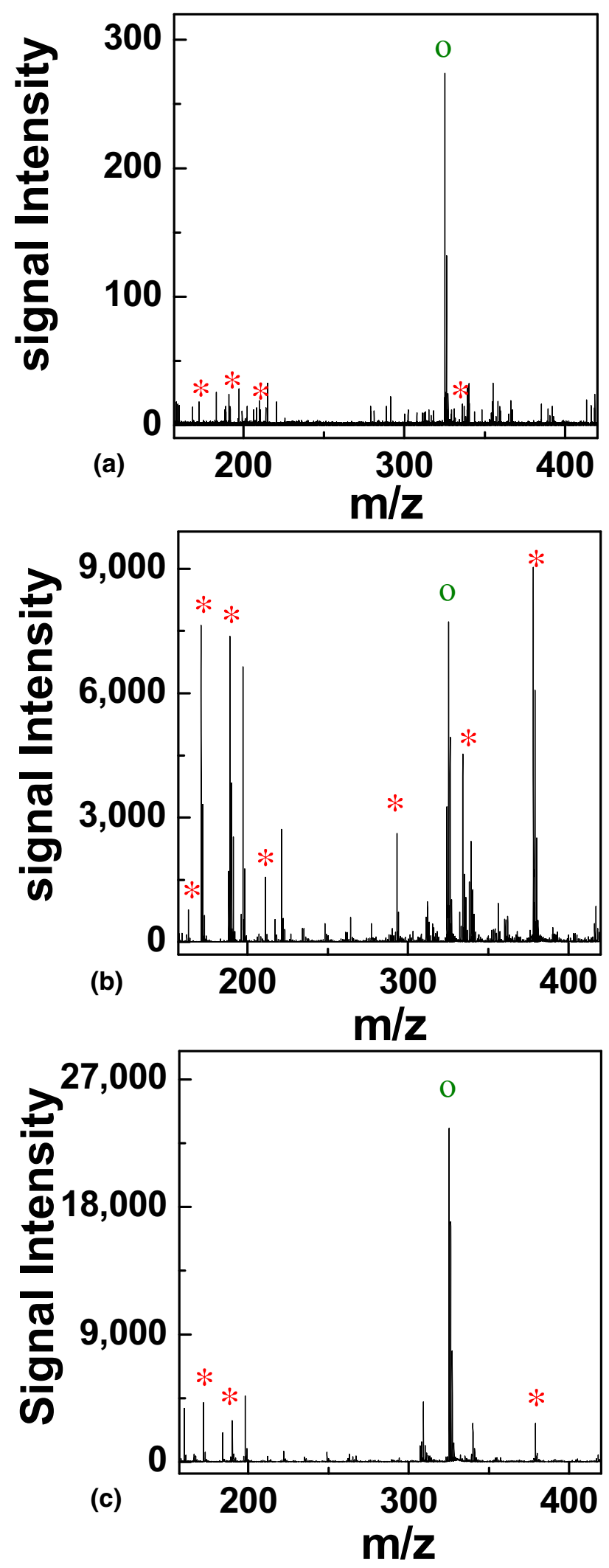

Figure 3. Representative MS spectra of 1 pmol quinidine collected using (a) CHCA/ANI in MALDI, (b) CHCA/ANI with $0.1 \%$ TFA in MALDI, and (c) CHCA/ANI in ME-SALDI. The matrix and analyte peaks were labeled with asterisks and circles, respectively.
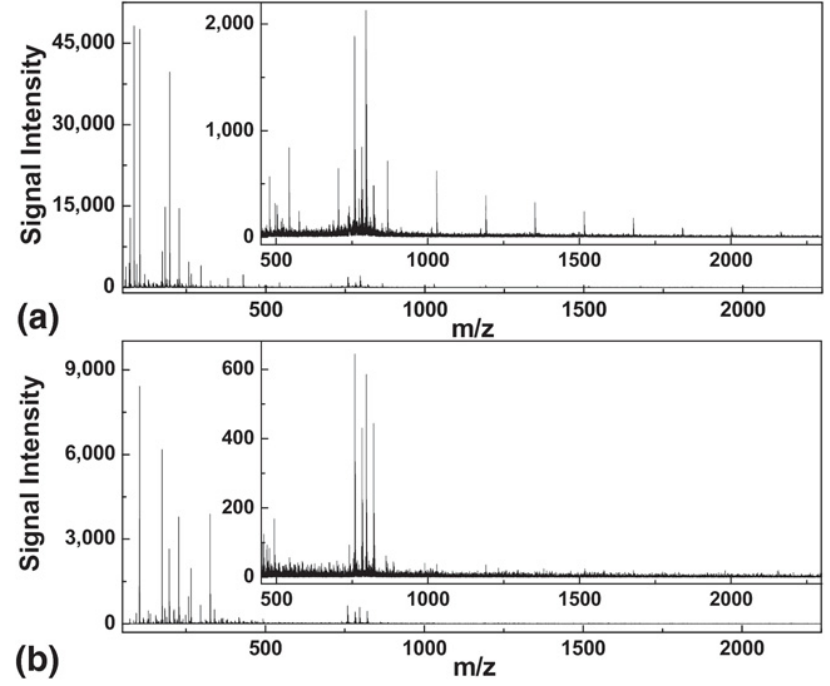

Figure 4. MS detection of garlic extracts using CHCA/ANI as the matrix in (a) ME-SALDI and (b) MALDI.

was utilized in the ME-SALDI mode. Similar improvement was observed when a different ionic matrix, $\mathrm{CHCA} / \mathrm{Py}$, was used as the ME-SALDI matrix in comparison with conventional MALDI, direct laser desorption ionization (LDI), and SALDI during the detection of garlic extract (Table 1).

The use of ionic matrix in ME-SALDI MS imaging in revealing the spatial distribution of natural biomolecules was demonstrated in Figure 5. An optical image of a $10-\mu \mathrm{m}$ thick stained garlic tissue section was shown in panel 5a where the storage leaf (i.e., endosperm) was clearly distinguishable from the cotyledon in the center. A piece of garlic section at the adjacent position was placed on a porous silicon substrate and coated with a thin-layer of CHCA/ANI through sublimation, followed by scanning with a $35-\mu \mathrm{m}$ laser beam at $50-\mu \mathrm{m}$ step wise. A major matrix ion $(m / z$ 190.1) was uniformly observed across the entire scanning area, which confirmed homogenous matrix deposition on the tissue and negligible matrix loss over the $\sim 46 \mathrm{~h}$ scanning (Figure $5 b)$. In comparison with the distorted distribution in DHB-coated garlic section (Figure 1b), the MS signal for the ions at $m / z 104.1$ was the same for the spectra acquired at the beginning and near the end of the tissue scanning. The much higher ion intensities observed in the cotyledon portion can therefore be assigned to local over-expression of $\gamma$-aminobutyric acid with reasonable confidence (Figure 5c). On the other hand, the potassium adducts of fructo-oligosaccharide DP5 $(\mathrm{m} / \mathrm{z}$ 867.2), one of the primary carbohydrate storage species in garlic, was found to distribute mainly in the endosperm portion, as expected (Figure $5 \mathrm{~d}$ ). Note that similar to the conventional matrix-based ME-SALDI MSI experiments [6], a lower laser fluence was used than that in MALDI MSI but yielded similar ion intensities (data not shown). This observation confirms that the presence of the tissue did not prevent the porous substrate underneath from 

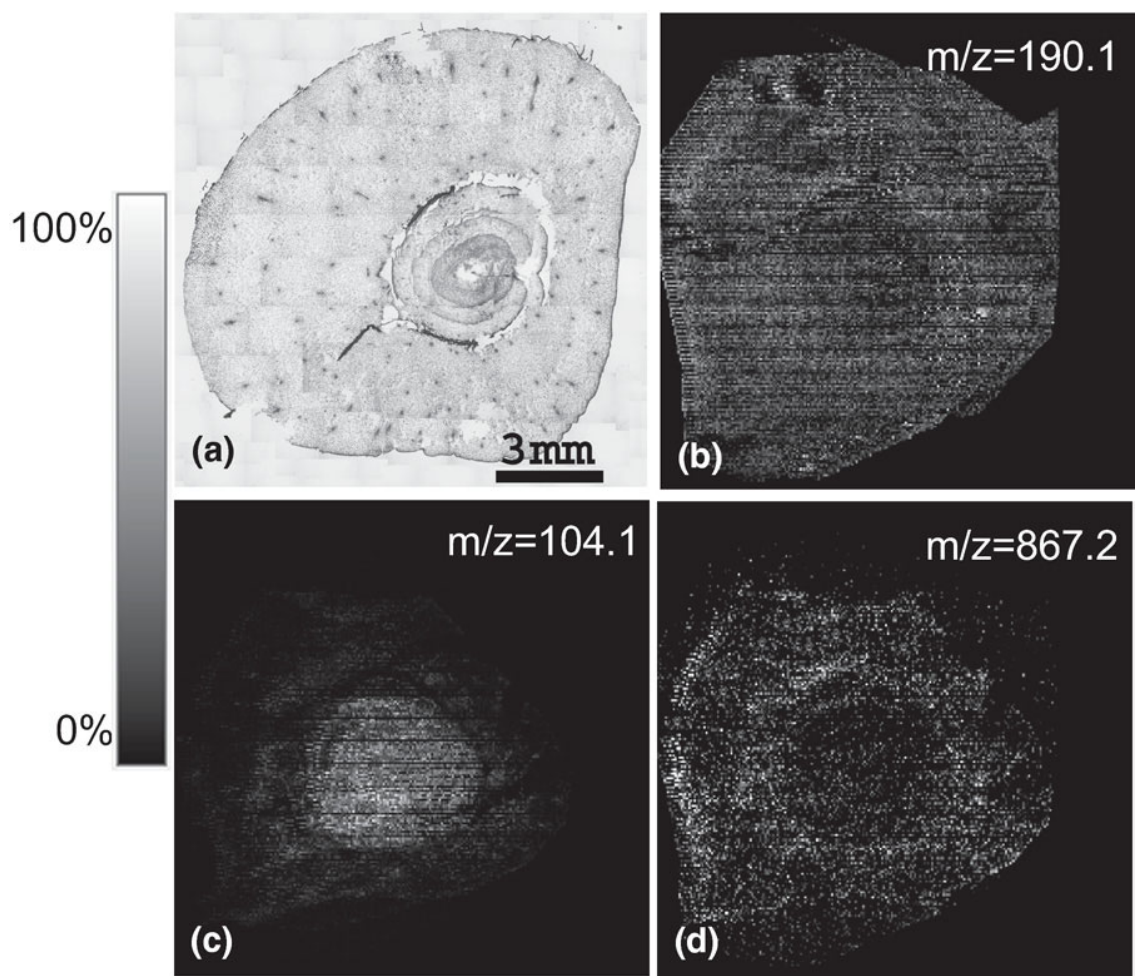

Figure 5. (a) An optical image of 10- $\mu \mathrm{m}$ thick garlic section. Reconstructed 2-D images for ions at (b) $m / z=190.1$, (c) $m / z=104.1$, and (d) $m / z=867.2$ from CHCA/ANI-coated garlic section in ME-SALDI IMS. For details see the text.

participating in the desorption and ionization process, which is consistent with our previous speculation that ME-SALDI is a combined thermo/mechanical process where ablation takes a minor role in analyte desorption [26].

\section{Conclusions}

In summary, we have demonstrated that the use of ionic matrix in ME-SALDI provides an attractive approach to the analysis of small molecules in the imaging application. The demonstrated benefits include extended imaging stability under vacuum, enhanced analyte ionization, and lower background noise. Elimination of acidic additives for ionic matrix without compromising analyte ionization efficiency makes it suitable for solvent-free matrix deposition. Qualitative mapping of low-mass species using ionic matrix in ME-SALDI has been demonstrated, and continuing studies on quantitative imaging are underway.

\section{Acknowledgments}

The authors thank Dr. Dykstra at the Laboratory for Advanced Electron and Light Optical Methods at College of Veterinary Medicine, North Carolina State University (NCSU) for tissue preparation. The authors acknowledge use of the optical microscope in Dr. Harold Ade's laboratory (Physics, NCSU).

\section{Appendix A Supplementary Material}

Supplementary material associated with this article may be found in the online version at doi:10.1016/ j.jasms.2009.08.011.

\section{References}

1. Dattelbaum, A. M.; Iyer, S. Surface-Assisted Laser Desorption/Ionization Mass Spectrometry. Expert Rev. Proteom. 2006, 3(1),153-161.

2. Northen, T. R.; Lee, J. C.; Hoang, L.; Raymond, J.; Hwang, D. R.; Yannone, S. M.; Wong, C. H.; Siuzdak, G. A Nanostructure-Initiator Mass Spectrometry-Based Enzyme Activity Assay. Proc. Natl. Acad. Sci. U.S.A. 2008, 105(10), 3678-3683.

3. Zhang, H.; Cha, S.; Yeung, E. S. Colloidal Graphite-Assisted Laser Desorption/Ionization MS and $\mathrm{MS}^{\mathrm{n}}$ of Small Molecules. 2. Direct Profiling and MS Imaging of Small Metabolites from Fruits. Anal. Chem. 2007, 79(17), 6575-6584.

4. Taira, S.; Sugiura, Y.; Moritake, S.; Shimma, S.; Ichiyanagi, Y.; Setou, M. Nanoparticle-Assisted Laser Desorption/Ionization Based Mass Imaging with Cellular Resolution. Anal. Chem. 2008, 80(12), 4761-4766.

5. Liu, Q.; Guo, Z.; He, L. Mass Spectrometry Imaging of Small Molecules Using Desorption/Ionization on Silicon. Anal. Chem. 2007, 79(10), 35353541 .

6. Liu, Q.; Xiao, Y.; Pagan-Miranda, C.; Chiu, Y. M.; He, L. Metabolite Imaging Using Matrix-Enhanced Surface-Assisted Laser Desorption/ Ionization Mass Spectrometry (ME-SALDI-MS). J. Am. Soc. Mass Spectrom. 2009, 20(1), 80-88.

7. Hankin, J. A.; Barkley, R. M.; Murphy, R. C. Sublimation as a Method of Matrix Application for Mass Spectrometric Imaging. J. Am. Soc. Mass Spectrom. 2007, 18(9), 1646-1652.

8. Armstrong, D. W.; Zhang, L. K.; He, L.; Gross, M. L. Ionic Liquids as Matrixes for Matrix-Assisted Laser Desorption/Ionization Mass Spectrometry. Anal. Chem. 2001, 73(15), 3679-3686.

9. Tholey, A.; Heinzle, E. Ionic (Liquid) Matrices for Matrix-Assisted Laser Desorption/Ionization Mass Spectrometry-Applications and Perspectives. Anal. Bioanal. Chem. 2006, 386(1), 24-37. 
10. Tu, T.; Sauter, A. D., Jr.; Sauter, A. D., 3rd; Gross, M. L. Improving the Signal Intensity and Sensitivity of MALDI Mass Spectrometry by Using Nanoliter Spots Deposited by Induction-Based Fluidics. J. Am. Soc. Mass Spectrom. 2008, 19(8), 1086-1090.

11. Carda-Broch, S.; Berthod, A.; Armstrong, D. W. Ionic Matrices for Matrix-Assisted Laser Desorption/Ionization Time-of-Flight Detection of DNA Oligomers. Rapid Commun. Mass Spectrom. 2003, 17(6), 553-560.

12. Li, Y. L.; Gross, M. L. Ionic-Liquid Matrices for Quantitative Analysis by MALDI-TOF Mass Spectrometry. J. Am. Soc. Mass Spectrom. 2004, 15(12), 1833-1837.

13. Mank, M.; Stahl, B.; Boehm, G. 2,5-Dihydroxybenzoic Acid Butylamine and Other Ionic Liquid Matrixes for Enhanced MALDI-MS Analysis of Biomolecules. Anal. Chem. 2004, 76(10), 2938-2950.

14. Jones, J. J.; Batoy, S. M.; Wilkins, C. L.; Liyanage, R.; Lay, J. O., Jr. Ionic Liquid Matrix-Induced Metastable Decay of Peptides and Oligonucleotides and Stabilization of Phospholipids in MALDI FTMS Analyses. J. Am. Soc. Mass Spectrom. 2005, 16(12), 2000-2008.

15. Zabet-Moghaddam, M.; Heinzle, E.; Lasaosa, M.; Tholey, A. Pyridinium-Based Ionic Liquid Matrices Can Improve the Identification of Proteins by Peptide Mass-Fingerprint Analysis with Matrix-Assisted Laser Desorption/Ionization Mass Spectrometry. Anal. Bioanal. Chem. 2006, 384(1), 215-224.

16. Hurtado, P.; Hortal, A. R.; Martínez-Haya, B. Matrix-Assisted Laser Desorption/Ionization Detection of Carbonaceous Compounds in Ionic Liquid Matrices. Rapid Commun. Mass Spectrom. 2007, 21(18), 3161-3164.

17. Laremore, T. N.: Zhang, F.; Linhardt, R. J. Ionic Liquid Matrix for Direct UV-MALDI-TOF-MS Analysis of Dermatan Sulfate and Chondroitin Sulfate Oligosaccharides. Anal. Chem. 2007, 79(4), 1604-1610.

18. Fukuyama, Y.; Nakaya, S.; Yamazaki, Y.; Tanaka, K. Ionic Liquid Matrixes Optimized for MALDI-MS of Sulfated/Sialylated/Neutral
Oligosaccharides and Glycopeptides. Anal. Chem. 2008, 80(6), 2171-2179.

19. Schnoell-Bitai, I.; Ullmer, R.; Hrebicek, T.; Rizzi, A.; Lacik, I. Characterization of the Molecular Mass Distribution of Pullulans by MatrixAssisted Laser Desorption/Ionization Time-of-Flight Mass Spectrometry Using 2,5-Dihydroxybenzoic Acid Butylamine (DHBB) as Liquid Matrix. Rapid Commun. Mass Spectrom. 2008, 22(19), 2961-2970.

20. Lemaire, R.; Tabet, J. C.; Ducoroy, P.; Hendra, J. B.; Salzet, M.; Fournier I. Solid Ionic Matrixes for Direct Tissue Analysis and MALDI Imaging. Anal. Chem. 2006, 78(3), 809-819.

21. Chan, K.; Lanthier, P.; Liu, X.; Sandhu, J. K.; Stanimirovic, D.; Li, J. MALDI Mass Spectrometry Imaging of Gangliosides in Mouse Brain Using Ionic Liquid Matrix. Anal. Chim. Acta 2009, 639(1/2), 57-61.

22. Finkel, N. H.; Prevo, B. G.; Velev, O. D.; He, L. Ordered Silicon Nanocavity Arrays in Surface-Assisted Desorption/Ionization Mass Spectrometry. Anal. Chem. 2005, 77, 1088-1095.

23. Bai, Q.; Fan, G.; Gu, Z.; Cao, X.; Gu, F. Effects of Culture Conditions on $\gamma$-Aminobutyric Acid Accumulation During Germination of Foxtail Millet (Setaria italica L.). Eur. Food Res. Technol. 2008, 228(2), 169-175.

24. Popov, V. N.; Eprintsev, A. T.; Fedorin, D. N.; Fomenko, O. Y. Igamberdiev, A. U. Role of Transamination in the Mobilization of Respiratory Substrates in Germinating Seeds of Castor Oil plants. Appl. Biochem. Microbiol. 2007, 43(3), 341-346.

25. Earle, M. J.; Esperanca, J. S. S.; Gilea, M. A.; Canongia Lopes, J. N.; Rebelo, L. P. N.; Magee, J. W.; Seddon, K. R.; Widegren, J. A. The Distillation and Volatility of Ionic Liquids. Nature 2006, 439, 831-834.

26. Xiao, Y.; Retterer, S. T.; Thomas, D. K.; He, L. Impacts of Surface Morphology on Ion Desorption and Ionization in Desorption Ionization on Porous Silicon (DIOS) Mass Spectrometry. J. Phys. Chem. C 2009, 113, 3076-3083. 